\title{
Experimental studies on the possible role of nitric oxide (NO) in the adaptogenic effects of Withania somnifera in rats
}

\author{
Nishant Rai ${ }^{1}$, Arunabha Ray $^{2}$, Kavita Gulati $^{3}$

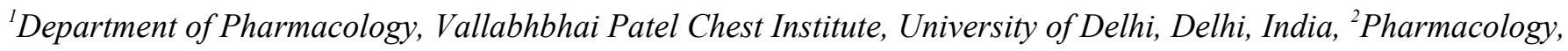 \\ Hamdard Institute of Medical Sciences, India, ${ }^{3}$ Vallabhbhai Patel Chest Institute, Delhi, India
}

Background: Adaptogens are agents primarily of herbal origin which help in coping with and/or attenuating stress effects and stress-related disorders. Withania somnifera (WS, Ashwagandha) is a classical medicinal plant in Indian traditional systems of medicine (Ayurveda and Unani) with adaptogenic properties and whose roots and aerial parts have been effectively used. The mechanisms of such effects are, however, not clearly defined. Nitric oxide (NO) is a multifunctional, signaling molecule with a wide array of physiological and pharmacological effects, and our earlier studies unequivocally demonstrated an important role for NO during stress. The present study thus investigated the possible role of NO in the anti-stress effects of WS extract in rats.

Methods: Restraint stress (RS) was used as the experimental stressor and the effects of WS pretreatments and their interactions with NO were assessed on stress-induced neurobehavioral, immunological and cellular/molecular markers, after chronic RS (x14).

Results: Chronic RS induced anxiogenic responses in the elevated plus maze test with reduced open arm entries / time after RS and WS pretreatment attenuated this response. Concurrent treatment with the NOS inhibitor, 7-nitroindazole, reversed the anti-anxiety effects of WS, whereas, sub-effective doses of NO mimetic (L-arginine) potentiated WS induced anxiolysis. WS treatment also induced elevations in NOx (stable NO metabolites) and reductions in MDA and ADMA levels in brain homogenates. In rats immunized with KLH, RS induced suppressions in both humoral and cell mediated immune responses, which were attenuated by WS treatment. Stress induced elevations in levels of pro-inflammatory cytokines (TNF-alpha and IL-6) and reductions in IL-4 and IFN-gamma levels were also differentially affected by WS pretreatment. These immunological changes were accompanied by elevations in plasma MDA and ADMA and lowered NOx levels. These immunological and biochemical changes seen after WS were reversed after L-NAME pretreatment, whereas, L-arginine potentiated the stress ameliorating effects of WS. Molecular studies showed that NOS gene expression in brain and blood were differentially influenced by RS and WS or NO modulators induced significant alterations in these RS effects.

Conclusions: These results suggest that NO mediated signaling mechanisms may be involved in the adaptogenic effects of WS. 